\title{
Rabaska
}

Revue d'ethnologie de l'Amérique française

\section{Audet, VéroniQue. Innu nikamu - L'Innu chante. Pouvoir des chants, identité et guérison chez les Innus. Québec, Les Presses de l’Université Laval, 2012, 292 p. ISBN 978-2-7637-9617-8}

\section{Bertrand Bergeron}

Volume 11, 2013

URI : https://id.erudit.org/iderudit/1018530ar

DOI : https://doi.org/10.7202/1018530ar

Aller au sommaire du numéro

Éditeur(s)

Société québécoise d'ethnologie

ISSN

1703-7433 (imprimé)

1916-7350 (numérique)

Découvrir la revue

Citer ce compte rendu

Bergeron, B. (2013). Compte rendu de [AUDET, VÉRONIQUE. Innu nikamu -

L'Innu chante. Pouvoir des chants, identité et guérison chez les Innus. Québec,

Les Presses de l'Université Laval, 2012, 292 p. ISBN 978-2-7637-9617-8].

Rabaska, 11, 183-187. https://doi.org/10.7202/1018530ar d'utilisation que vous pouvez consulter en ligne. 


\section{Comptes rendus}

Audet, VéRONIQUE. Innu nikamu - L'Innu chante. Pouvoir des chants, identité et guérison chez les Innus. Québec, Les Presses de l'Université Laval, 2012, 292 p. ISBN 978-2-7637-9617-8.

À Mashteuiatsh, où réside une importante communauté montagnaise, innu se prononce ilnu. M'enquérant de cette disparité phonétique auprès de Louise Siméon qui travaille au musée amérindien de l'endroit, celle-ci m'apprit qu'au Lac-Saint-Jean, les Montagnais parlaient en « $1 »$ alors que sur la Côte-Nord, ils employaient le « $\mathrm{n} »$. Cette réponse me satisfit d'autant plus qu'elle réveillait en moi mon apprentissage de l'alphabet grec dans lequel les consonnes «l,m,n,r» $(1, m, n, r)$ copinaient entre elles sous l'appellation de consonnes liquides. Il y avait bien parenté, mais pas au point de s'étendre jusqu'aux langues indo-européennes dont l'ilnu ne fait évidemment pas partie.

À Mashteuiatsh, la langue ilnue n'est presque plus en usage comme à Essipit d'ailleurs (p. 169) aux dires de Véronique Audet dans son essai fouillé, Innu nikamu - L'Innu chante. Pouvoir des chants, identité et guérison chez les Innus. Le titre, à lui seul, est suffisamment explicite pour décrire la problématique abordée : quel rôle attribuer aux chants dans la valorisation de l'identité et le processus de guérison personnelle et sociale des douze communautés innues dispersées le long de la rive nord du Saint-Laurent jusqu'au Labrador? Seules les communautés de Mashteuiatsh et de Matimekush-Lac-John vivent dans des réserves situées au cœur du territoire québécois.

Véronique Audet a limité son terrain d'enquête aux populations vivant dans les réserves "d'Ekuanitshit (Mingan) et de Uashat mak Mani-utenam (Sept-Îles et Maliotenam) de juillet à octobre 2003 » (p. 20). Cependant, ses contacts remontent à 1997. « Mais de façon plus diffuse [... son] expérience de "terrain" s'étend de juin 2003 à août 2005 pour la réalisation du présent ouvrage et se poursuit toujours » (p. 21). Elle qualifie sa méthode d'enquête d' " observation participante ». Véronique Audet n'est pas de ces chercheurs qui revendiquent la « self-elemination » dans leur pratique. Elle privilégie l'implication personnelle au nom d'une compréhension empathique et holistique. Celle-ci « est non seulement observation de ce qui se passe autour de nous, mais aussi, et surtout, un engagement, dans le champ des relations sociales et culturelles, voire spirituelles [...] de la part de l'ethnographeanthropologue. C'est une participation consciente, systématisante, réflexive, 
éthique et émotive » (p. 22). En somme, toute la personnalité de la chercheuse est engagée dans la cueillette de ses informations.

En suscitant, par cette méthode, l'amitié de ses informateurs, elle était en mesure de recueillir des témoignages qui traduisent en profondeur « l'être au monde »-expression qui revient fréquemment sous sa plume - des communautés qu'elle a fréquentées. Pour heuristique qu'elle soit, cette méthode n'en possède pas moins ses limites liées à l'équation personnelle de la chercheuse. Véronique Audet n'en fait pas mystère. C'est une intellectuelle blanche, de sexe féminin, mère d'une fille qui partageait ses séjours sur le terrain. Elle possède une connaissance limitée de la langue innue sans être vraiment musicienne (p. 21).

Son essai se divise en quatre chapitres précédés d'une introduction élaborée et suivis d'une conclusion, d'une bibliographie détaillée (une médiagraphie en fait), d'un résumé et d'un petit lexique innu fort utile. Louons la délicatesse de l'auteur d'avoir accompagné son livre d'un CD renfermant vingt-trois chansons. Les matériaux d'enquête nous sont, en partie, rendus accessibles.

L' «Introduction », élaborée et étoffée, situe la démarche de la chercheuse à partir de trois ancrages : personnel (p. 1), social (p. 4) et anthropologique (p. 7). Nous avons abordé, plus haut, les implications de cette posture ethnographique.

Le « chapitre 1 » adopte une perspective diachronique qui permet de situer la pratique actuelle de la musique et du chant innus en regard de ses origines. Il en ressort que le son est très important dans la culture innue. Il constitue, en quelque sorte, une interface entre le monde humain et non humain en vertu de sa double nature qui en fait un passeur par excellence : le son est à la fois matériel par les ondes sonores mises en branle par un instrument ou par la bouche, et immatériel par le choc émotionnel qu'il engendre par son audition. Le tambour (teueikan), instrument emblématique de la culture traditionnelle, est à la fois émetteur et capteur de son. Sa forme circulaire évoque le cosmos et sa membrane est fabriquée en peau de caribou, animal lui aussi emblématique de cette nation. En jouer dresse une frontière entre ce monde-ci et un autre monde, comme si chacun de ces univers se concentrait de part et d'autre de la membrane pour communiquer à travers ce qui les sépare, élevant le tambour au rang d'un gigantesque tympan cosmique. Cette conception du teueikan explique le respect dont on l'entoure. Le tambour accompagne le chant. Celui que le chanteur recevait en rêve était sa propriété personnelle. « [S]a signification ne doit pas être révélée ou expliquée, sans quoi [son] pouvoir s'affaiblit. C'est pourquoi les textes sont marmonnés et indéchiffrables pour la majorité des Innus. Ils sont chantés dans une langue ésotérique propre au domaine linguistique du chant, reconnu comme étant le langage des esprits » 
(p. 69). Le chant, le teueikan et la danse lors d'un makusan (festin de graisse de caribou), que certains ont comparé à l'eucharistie (p. 74), forment une triade essentielle au maintien de la cohésion sociale innue.

Si le premier chapitre s'emploie à tracer un portrait traditionnel de la culture musicale innue, le deuxième tente d'en saisir l'évolution dans le temps. La société innue n'est pas fermée aux influences des courants culturels étrangers que les médias de masse répandent à travers la planète. Pour éviter de sombrer dans l'immense océan sonore de la musique occidentale, les artistes innus se sont approprié les diverses formes qui l'incarnent. Cette appropriation est à la fois résistance et accueil par « indigénisation ». L'auteur établit la chronologie de l'introduction des musiques étrangères : « les chants chrétiens, les pièces pour violon, accordéon, harmonica, guimbarde, etc. ; le country; le rock, le folk; les musiques (néo) traditionnelles panamérindiennes ou de pow-wow ; enfin, les divers genres tels que le reggae, le hip-hop/rap, le heavy métal, etc. » (p. 83).

Les " chapitres 3 et 4 » présentent un point de vue synchronique des problèmes identitaires et des blessures personnelles et sociales des membres de la nation innue. Ainsi que l'avait laissé entrevoir l' «Introduction », la société innue vit une crise au sens où l'entendait le philosophe Gramsci : le vieux n'arrive pas à disparaître et le neuf ne parvient pas à apparaître. Les individus se sentent coincés entre les deux, embourbés dans une double contrainte : les aînés ne peuvent plus transmettre leur culture en situation de chasse, de pêche et de cueillette à cause des transformations sociales subies, et ils ne peuvent pas adopter la culture du colonisateur au risque de perdre leur mode d'être au monde. Dans une situation où l'individu ne peut ni fuir ni affronter, disait Henri Laborit, il est inévitablement acculé à l'inhibition de l'action. « Plusieurs jeunes sont devenus mésadaptés à la vie traditionnelle et tout autant mésadaptés à la vie allochtone » (p. 27). Il est normal, voire vital, que dans une situation qui semble inextricable, la culture traditionnelle devienne une valeur refuge. Mais s'y complaire devient un leurre mortel. La jeune génération et celle qui l'a précédée brandissent le chant comme un outil de revendication et de revalorisation/revitalisation identitaires. En ce sens, la langue devient un objet de respect et d'attention particuliers, car « [s]elon une conception populaire innue, la qualité de la langue est garante de la qualité de l'identité innue d'une personne » (p. 169). N'est-elle pas, après tout, le premier marqueur culturel et identitaire d'une nation ? On ne connaît pas de civilisation muette. Et la culture qu'elle véhicule devient à la fois une référence et une différence. Être Innu, dans le concert des nations, c'est ne pas être Français, Anglais, Allemand. Dans le même temps, au cœur d'une dialectique du même et de l'autre, le même trouve sa justification qui avalise 
le mode d'être au monde. La conscience de soi prend souvent naissance dans la prise de conscience de l'existence de l'autre qui nous résiste tout en nous permettant de nous affirmer.

Cette revalorisation identitaire ne peut faire l'impasse sur le mal-être personnel et social qui sévit à l'état endémique dans certaines collectivités. Le drame qui se vivait à Davis Inlet et qui fut porté à l'attention du grand public a servi de révélateur d'une crise à la fois sociale et identitaire qui secouait cette communauté innue. On ne peut s'empêcher d'observer un scénario de type rédemption dans les chants liés au thème de la guérison que l'auteur décortique au quatrième chapitre. Le schéma narratif de ces complaintes est assez conventionnel : aveu et reconnaissance du mal-être personnel, acceptation et volonté affirmée de s'en sortir. Plus les chansons traitent d'un problème personnel, plus elles touchent les gens de près par le mécanisme bien connu de la projection/identification. L'auditeur se reconnaît dans l'évocation du drame intime, créant ainsi une fraternité consensuelle de gens qui souffrent, donnant raison à Hugo qui admonestait son lecteur dans Les Contemplations : «Insensé qui crois que je ne suis pas toi !»

Les témoignages touchants et sincères abondent dans cette partie. Les paroliers affrontent leurs problèmes sans détour, l'expriment dans une langue simple et directe. La musique qui se greffe aux paroles finit par susciter cette catharsis espérée. La lecture des textes des chansons et des témoignages offre une réponse sans équivoque à cette question lancinante que doit, un jour ou l'autre, affronter tout créateur : «Faites-vous cet aveu : devriezvous mourir s'il vous était interdit d'écrire? » (Lettres à un jeune poète, Rainer Maria Rilke). On devine aisément que les artistes innus répondraient par l'affirmative. Les récents témoignages de la Commission vérité et réconciliation sur les exactions subies dans les pensionnats démontrent, si besoin en est, le pouvoir thérapeutique de la parole.

Dans une langue simple, éloignée de tout jargon hermétique, Véronique Audet réussit à nous faire partager de manière empathique les vicissitudes et les drames qui se vivent dans les communautés innues doublement aliénées sur le plan personnel et social, minorité évoluant au sein d'une nation, le Québec, qui, elle-même, n'a pas eu la détermination de régler son contentieux historique. Innu Nikamu nous en propose une vision en abîme. La démarche empathique de la chercheuse, son observation participante répondent au désir profond de ses informateurs devenus ses amis : « À quelques occasions, les Innus m'ont d'ailleurs affirmé que je ne pourrais vraiment comprendre leur réel vécu sans partager pleinement, accepter de les côtoyer, même de vivre moi-même leur vie, leur enivrement, leurs déroutes et leurs souffrances » (p. 216). Cet essai ne peut que susciter la sympathie de ses éventuels lecteurs. 
En prime, Véronique Audet nous offre ces chants qui ont nourri sa recherche, dans cette langue des bois façonnée par le mode d'être au monde particulier des Innus.

BERTRANd BERgeron

Saint-Bruno en Lac-Saint-Jean

Belmont, Nicole. Mythe, conte et enfance. Les écritures d'Orphée et de Cendrillon. Paris, L'Harmattan, «Anthropologie du monde occidental », 2010, 344 p. ISBN 978-2-296-12604-6.

Trois chantiers majeurs ont occupé la vie professionnelle de Nicole Belmont jusqu'à ce jour : les traditions diverses entourant la naissance, le questionnement sur le statut du folklore dont la disqualification est devenue un impensé de l'ethnologie française et l'étude des contes de tradition orale.

C'est aux résultats attribuables à ce troisième chantier que nous devons Mythe, conte et enfance. Les écritures d'Orphée et de Cendrillon. Le titre, pour détaillé qu'il soit, n'épuise pas la matière du livre, pas plus que la référence aux « écritures » ne renvoie exclusivement à la tradition lettrée. Bien au contraire. Cet essai ne propose pas une avancée nouvelle dans la pensée de l'auteur. Il s'agit plutôt d'une compilation d'articles qui récapitule les travaux de la chercheuse, témoignage " d'une quête de plus de vingt ans à travers les contes de tradition orale » (p. 7).

En incluant l'« Introduction » et la « Coda », vingt-quatre articles ont été rassemblés et regroupés en trois parties : «Les contes déménagent : de l'oral à l'écrit, et retour » (six articles), « Des contes pour enfants ? » (six articles) et « Contes merveilleux » (dix articles). Quant à l' «Introduction » (p. 13), elle traite de la « [r] echerche du sens en ethnologie de l'Europe et en folklore », alors que la « Coda » examine les « lacunes, altérations, lapsus dans le récit oral» (p. 327). Tous ces articles ont fait l'objet d'une publication dans l'une ou l'autre des revues spécialisées concernées de près ou de loin par les sujets traités : Cahiers de littérature orale, Topique, L'Homme, Le Journal des anthropologues, Ethnologie française, etc.

Le nombre d'articles rend impossible la tâche de faire le compte rendu de chacun, aussi m'emploierai-je à tracer les lignes de force qui traversent cette compilation plus que bienvenue dans le monde des études sur le conte de tradition orale. Comme l'auteur poursuit la même réflexion d'un article à l'autre à travers des thèmes connexes, il n'est pas étonnant qu'elle reprenne, quand besoin est, ses propos dans les mêmes termes. Il faut voir dans ce retour du même le constat que l'expression de ses idées a atteint à ce moment-là 